\title{
Successful vaginal delivery of spontaneously conceived twins in each horn of a bicornuate uterus with previous caesarean: a case report
}

\author{
Farhat Mazhari*, Arpita De, Reva Tripathi
}

Department of Obstetrics and Gynaecology, Hamdard Institute of Medical Sciences and Research and HAHC Hospital, New Delhi, India

Received: 21 August 2019

Accepted: 30 September 2019

\author{
*Correspondence: \\ Dr. Farhat Mazhari, \\ E-mail: farhatmazhari1987@gmail.com
}

Copyright: () the author(s), publisher and licensee Medip Academy. This is an open-access article distributed under the terms of the Creative Commons Attribution Non-Commercial License, which permits unrestricted non-commercial use, distribution, and reproduction in any medium, provided the original work is properly cited.

\begin{abstract}
Mullerian duct fusion anomalies resulting in uterine malformations have prevalence of 3-4\%. Among this, bicornuate uterus has a rare incidence of $0.4 \%$. Uterine malformations pose significant threat in terms of obstetric outcomes. We report an extremely rare case of spontaneous conception of twin pregnancy with the fetus occupying each horn of a bicornuate uterus (bicornis unicollis), in a woman with a history of previous caesarean section. She was booked at our hospital and the pregnancy remained uneventful. At 35 weeks she went into spontaneous labour and delivered vaginally without any maternal-fetal complications. The case is unique and the management is worth discussing as till date no protocols or guidelines have been proposed for the mode of delivery of bicornuate uterus with twins with previous cesarean. Only 12 cases of twins with bicornuate uterus have been reported till date. This is the first case in literature in which a successful VBAC has been conducted in a woman with bicornuate uterus with twins with previous caesarean.
\end{abstract}

Keywords: Bicornuate uterus, Mullerian anomalies, Twin pregnancy, Uterine malformations

\section{INTRODUCTION}

Failure of fusion of Mullerian ducts result into various types of uterine malformations. The actual incidence varies because many of the subclinical cases remain undiagnosed. The incidence of congenital uterine anomalies is approximately $5.5-6.7 \%$ among the general population, $5-10 \%$ in women with recurrent early pregnancy loss and about $25 \%$ in women with late first or second trimester pregnancy loss or preterm delivery. ${ }^{1-3}$ They are associated with worse obstetric outcomes such as miscarriage, preterm labour, premature rupture of membranes, malpresentations and third stage complications. Bicornuate uterus with a rare incidence of $0.4 \%$ is Type IV according to ASRM classification and U3 according to ESHRE classification. ${ }^{1,2,4}$ Incidence of twin pregnancies in patients with uterine anomalies is $2.7-3.1 \% .4,5$
Spontaneous twin gestation in a case of bicornuate uterus with one fetus occupying each uterine horn has a very rare incidence. We report a rare case of uterus bicornuate unicollis with spontaneous conception of twin pregnancy occupying each horn in a woman with a previous caesarean section. The pregnancy continued till 35 weeks and patient delivered both twins vaginally without any maternal-fetal complications. This highlights the need to individualise treatment and TOLAC may be considered in selected cases of bicornuate cases with previous cesarean. The case was unique because of its extremely rare incidence and paucity of a proper guideline for the management of such cases.

\section{CASE REPORT}

A 28-year-old second gravida, para 1 live 1 (G2P1L1), with spontaneous conception of dichorionic diamniotic 
(DCDA) twins with bicornuate uterus presented to the antenatal outpatient department at $22+4$ weeks, for booking. She was married for four years and had a previous caesarean section around three years back in view of bicornuate uterus with breech presentation. On clinical examination, uterus was 24 weeks size, two separate horns could be palpated per abdomen. On per speculum examination, single cervix was seen. The woman had her first ultrasonography at 9+6 weeks, showing a bicornuate uterus with twin gestational sacs with live fetus in both horns (Figure 1). The second scan at 14+2 weeks showed normal nuchal translucency and nasal bone presence in both twins (Figure 2). Cervical length was normal. Level II scan ruled out congenital anomalies in both twins. All routine antenatal investigations were normal. She was found to be hypothyroid at her first visit and started on thyroxin tablets. She followed up at monthly intervals and her clinical examinations were normal. Repeated scans were done at monthly intervals for growth monitoring and cervical length. Liquor volume and growth parameters were adequate for gestational age and no discordance was seen.

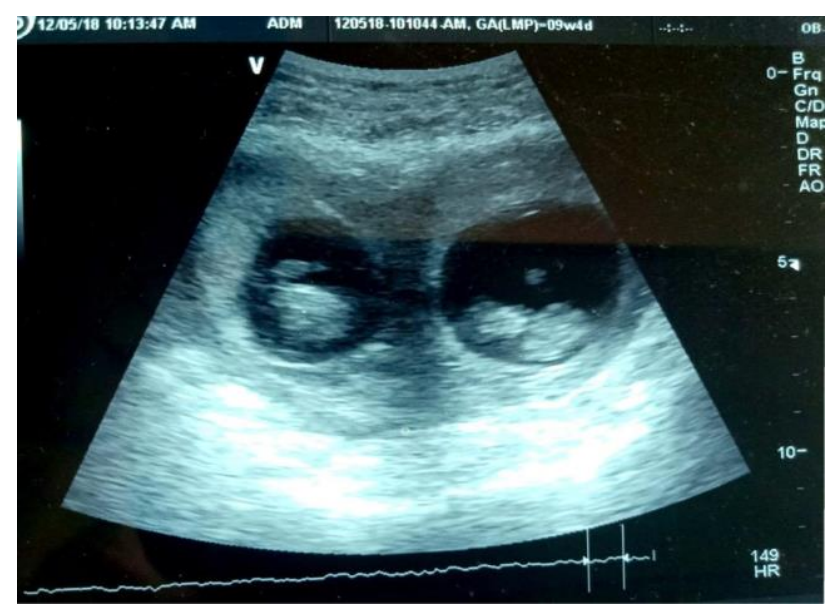

Figure 1: Ultrasound at 9+6 weeks showing bicornuate uterus with twin pregnancy in each cornua.

At gestational age of $35+2$ weeks, she presented to labour room emergency with labour pains and leaking per vaginum. Her vitals were normal with pulse of 80 beats/min and blood pressure of 110/80 $\mathrm{mmHg}$. On per abdominal examination, an overdistended uterus with both horns was palpable, multiple fetal parts were felt, both were in cephalic presentation and two distinct fetal heart sounds were heard. Clinically effective fetal weight was estimated to be around $2 \mathrm{~kg}$ for both fetuses. Two contractions, lasting for ten to fifteen seconds were elicited in ten minutes. Per speculum examination showed clear leaking per vaginum. On per vaginal examination, cervical os was $2 \mathrm{~cm}$ dilated, $50 \%$ effaced, membranes present but with leaking present, and vertex of first twin at -2 station. Pelvic assessment showed adequate pelvis.

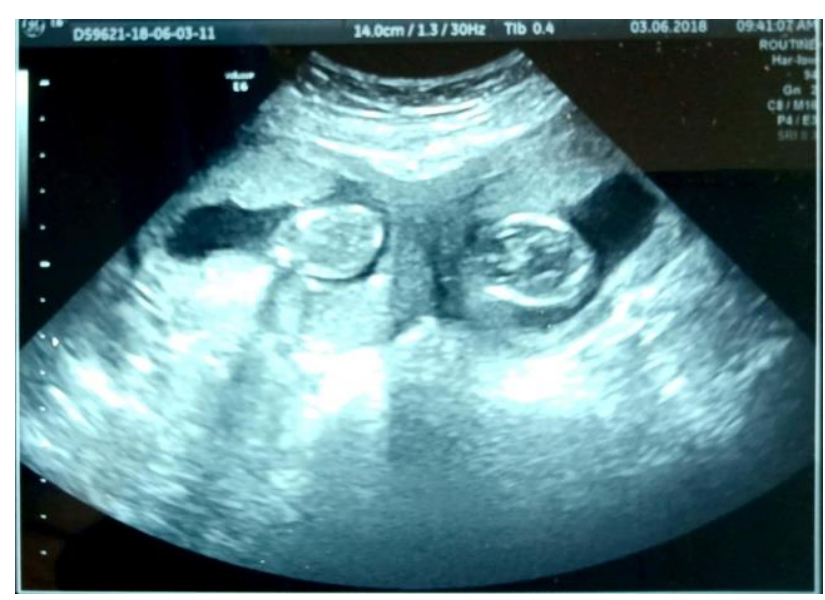

Figure 2: Ultrasound at $14+2$ weeks showing bicornuate uterus with DCDA twins.

Considering preterm babies with preterm premature rupture of membranes (PPROM) but with good Bishops score and spontaneous onset of labour, she was given Trial of Labour After Caesarean (TOLAC). Labour was augmented with oxytocin starting with a dose of 2 $\mathrm{Miu} / \mathrm{ml}$ and escalated till $4 \mathrm{Miu} / \mathrm{ml}$. Vigilant monitoring of patient's pulse rate, contractions and continuous fetal heart rate monitoring by CTG was done. Her first stage lasted for four hours. Second stage lasted for one and a half hours. In the pushing phase of second stage, when the vertex was almost at the outlet, the fetal heart rate of the first twin was dipping to 80 beats/min lasting for almost one minute. Forceps assisted vaginal delivery of first twin was done followed by spontaneous delivery of second twin after 12 minutes. Two separate placentas were removed. The first baby was a female child of 1.9 $\mathrm{kg}$ and the second baby was male of $2.05 \mathrm{~kg}$. Both had APGAR score of 10/10. Both babies were shifted to the mother. Postnatal period was uneventful. Patient was discharged on third day in a stable condition. She was followed up after six weeks in postnatal clinic and had no complaints. Both babies were doing fine and breastfeeding well. She was advised for progesterone pills and injectables as a contraceptive choice to which she refused. She was then counselled to use barrier methods as a contraceptive.

\section{DISCUSSION}

Mullerian duct fusion abnormalities lead to a wide variation of uterine anomalies. Bicornuate uterus is a type IV Mullerian fusion defect as classified by American Society of Reproductive Medicine (ASRM). It can be uterus bicornuate bicollis (double cervix) or uterus bicornuate unicollis (single cervix), as in this case. More recently in the year 2013, ESHRE/ESGE has classified uterine anomalies including cervical and vaginal anatomical variations (Figure 3). Bicorporeal uterus or class U3 is characterised by the presence of an external indentation on uterine fundus exceeding $50 \%$ of uterine wall thickness. ${ }^{6}$ The ESHRE classification of this case stands at U3COV0 (bicornuate uterus with normal single 
cervix and a normal vagina). This indentation can partly or completely divide the uterine cavities including cervix and vagina in some cases which will be accordingly reflected in the class as $\mathrm{U} 3 \mathrm{C} 1$ or $\mathrm{C} 2 \mathrm{~V} 1$ or V2. Diagnosis of uterine malformation is done by $2 \mathrm{D}$ or $3 \mathrm{D}$ multiplanar ultrasonography, hysterosalpingography, MRI or invasive methods like laparo-hysteroscopy. ${ }^{7}$

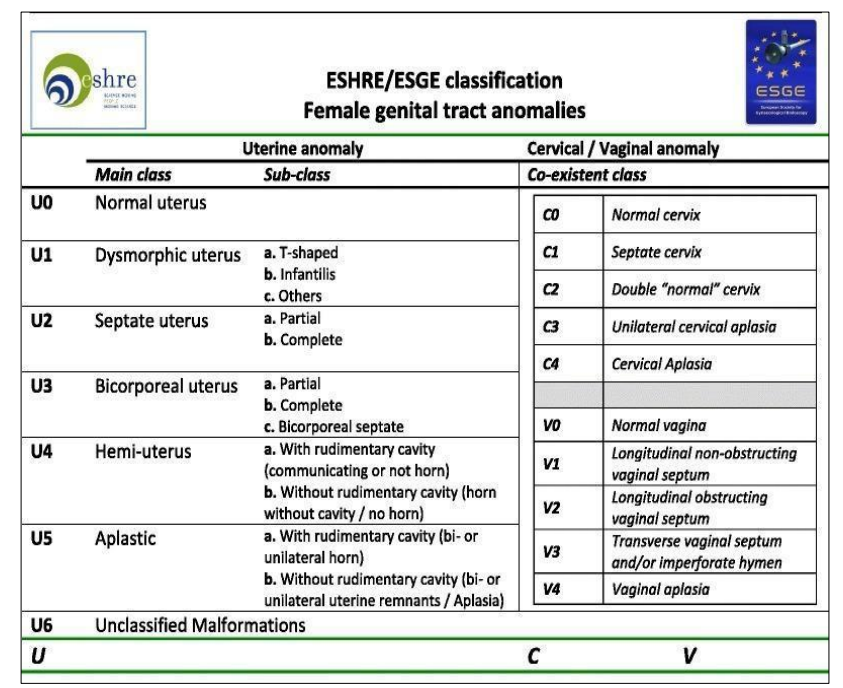

Figure 3: ESHRE/ESGE classification of female genital tract malformations.

Obstetric outcomes are poor in terms of recurrent miscarriages, intrauterine growth restriction, preterm labour, malpresentations, premature rupture of membranes, need for operative delivery, cervical dystocia, uterine rupture and third stage complications. ${ }^{8-10}$ Different theories like diminished muscle mass, abnormal uterine blood flow, and cervical insufficiency explain the associated obstetric outcome. ${ }^{11}$ In our case, the pregnancy progressed well till 35 weeks 2 days

Very few cases have been reported of women with twin pregnancies in uterine malformations, specifically bicornuate uterus. ${ }^{5}$ The complexity is increased by the previous cesarean scar. Till date only 12 cases have been reported of twin pregnancy in bicornuate uterus. ${ }^{12-16}$ With very few cases to report, there are no proper guidelines on mode of delivery of such patients.

Even though this was a preterm birth, the outcome has been good. This patient was given no progesterone supplements as the outcome of the previous pregnancy had been good. Some studies prove that use of vaginal progesterone and cervical cerclage in twin pregnancy cannot prevent preterm delivery. ${ }^{5,17,18}$ Fox et al's study on the use of cervical cerclage in twin pregnancies in the abnormal uterus showed no benefit. ${ }^{5}$ Risk benefit ratio can be considered for use of progesterone and cerclage according to case scenario. In patients with a bad history vaginal progesterones may be advised as it is not associated with many adverse side effects.
There are no specific guidelines on the mode of delivery in cases of twin pregnancy and bicornuate uterus. But the incidence of caesarean section is significantly higher in cases of uterine malformations because of the associated contractility abnormalities in labour and cervical dystocia. ${ }^{19,20}$ Rare incidence of uterine rupture during induction of labour in uterine malformations has also been reported. ${ }^{21}$

However, in our case, an individualized decision was taken in consultation with senior obstetricians. She was given a trial of labour for Vaginal Birth After Caesarean (VBAC) as she had PPROM, favorable Bishops score, and a smooth progress of labour with strict monitoring. No progesterone or cerclage was given in this case. Corticosteroids may be given to prevent complications of preterm delivery. This is the first case in literature with a bicornuate uterus with twin pregnancy with previous cesarean in which a successful VBAC was conducted. These outcomes prove that trial of VBAC can be given to patients having uterine malformations with previous cesarean provided the other criteria are met. However, more studies are required to actually put forward a proper guideline for the management and mode of delivery in such cases.

\section{CONCLUSION}

Incidence of uterine anomalies is low but the associated bad obstetric prognosis makes it significant to be diagnosed at an early stage for better management. Management of a patient with a twin pregnancy in a bicornuate uterus with history of previous caesarean section should be individualised. TOLAC may be considered if all pre-requisites of VBAC like clinical estimated fetal weight, favourable Bishops score, adequate pelvis and facilities for intensive monitoring are available. More studies are required to come up with specific guidelines in different scenarios.

\section{Funding: No funding sources \\ Conflict of interest: None declared \\ Ethical approval: Not required}

\section{REFERENCES}

1. Chan YY, Jayaprakasan K, Zamora J, Thornton JG, Raine-Fenning N, Coomarasamy A. The prevalence of congenital uterine anomalies in unselected and high-risk populations: a systematic review. Hum Reprod Update. 2011;17(6):761-71.

2. Saravelos SH, Cocksedge KA, Li TC. Prevalence and diagnosis of congenital uterine anomalies in women with reproductive failure: a critical appraisal. Hum Reprod Update. 2008;14(5):415-29.

3. Acién P. Incidence of Müllerian defects in fertile and infertile women. Hum Reprod. 1997;12(7):1372.

4. Fox NS, Roman AS, Saltzman DH, Klauser CK, Rebarber A. Twin pregnancy in patients with a 
uterine anomaly. J Matern Fetal Neo Med. 2014;27(4):360-4.

5. Heinonen PK. Twin pregnancy in the congenital malformed uterus. J Obstet Gynaecol. 2016;36(5):571-3.

6. Grimbizis GF, Gordts S, Di Spiezio Sardo A, Brucker S, De Angelis C, Gergolet M, et al. The ESHRE/ESGE consensus on the classification of female genital tract congenital anomalies. Hum Reprod. 2013;28(8):2032-44.

7. Bhagavath B, Greiner E, Griffiths KM, Winter T, Alur-Gupta S, Richardson $\mathrm{C}$, et al. Uterine malformations: an update of diagnosis, management, and outcomes. Obstet Gynecol Surv. 2017;72(6):377-92

8. Raga F, Bauset C, Remohi J, Bonilla-Musoles F, Sim'on C, Pellicer A. Reproductive impact of congenital M"ullerian anomalies. Hum Reprod, 1997;12(10):2277-81.

9. Cohen AW, Chhibber G. Obstetric complications of congenital anomalies of the paramesonephric ducts. Seminars Repro Endocrinol. 1986;4(1):59-65.

10. Green LK, Harris RE. Uterine anomalies. Frequency of diagnosis and associated obstetric complications. Obstet Gynecol. 1976;47(4):427-9.

11. Reichman DE, Laufer MR. Congenital uterine anomalies affecting reproduction. Best Pract Res Clin Obstet Gynaecol. 2010;24(2):193-208.

12. Arora M, Gupta N, Neelam N, Jindal S. Unique case of successful twin pregnancy after spontaneous conception in a patient with uterus bicornis unicollis. Arch Gynecol Obstet. 2007;276(2):193-5.

13. Cruceyra M, Iglesias C, la Calle M, Ssncha M, Magallon SL, Gonz'ales S. Successful delivery of a twin pregnancy in a bicornuate uterus (uterus bicornis unicollis) by bilateral Caesarean section. J Obstet Gynaecol Can. 2011;33(2):142-4.

14. Ahmad FK, Sherman SJ, Hagglund KH. Twin gestation in a woman with a uterus didelphys: a case report. J Reprod Med. 2000;45(4):357-9.
15. Jones MM, Flanagan MC. Twin pregnancy in a uterus didelphys delivered by bilateral repeat cesarean sections. J Natl Med Assoc. 1973;65(1):534.

16. Kekkonen R, Nuutila M, Laatikainen T. Twin pregnancy with a fetus in each half of a uterus didelphys. Acta Obstet Gynecol Scand. 1991;70(45):373-4.

17. Rode L, Klein K, Nicolaides K, Krampl-Bettelheim E, Tabor A. Prevention of preterm delivery in twin gestations (PREDICT): a multicenter, randomized, placebo-controlled trial on the effect of vaginal micronized progesterone. Ultrasound Obstet Gynecol. 2011;38(3):272-80.

18. Saccone G, Rust O, Althuisius S, Roman A, Berghella V. Cerclage for short cervix in twin pregnancies: systematic review and meta-analysis of randomized trials using individual patient-level data. Acta Obstet Gynecol Scand. 2015;94(4):352-8.

19. Zhang Y, Zhao Y, Qiao J. Obstetric outcome of women with uterine anomalies in China. Chin Med J(Engl). 2010;123(4):418-22.

20. Shuiqing M, Xuming B, Jinghe L. Pregnancy and its outcome in women with malformed uterus. Chin Med Sci J. 2002;17(4):242-5.

21. Ravisia DJ, Brain PH, Pollard JK. Incidence of uterine rupture among women with mullerian duct anomalies who attempt vaginal birth after cesarean delivery. Am J Obstet Gynecol. 1999;181(4):877-81.

Cite this article as: Mazhari F, De A, Tripathi R. Successful vaginal delivery of spontaneously conceived twins in each horn of a bicornuate uterus with previous caesarean: a case report. Int J Reprod Contracept Obstet Gynecol 2019;8:4581-4. 